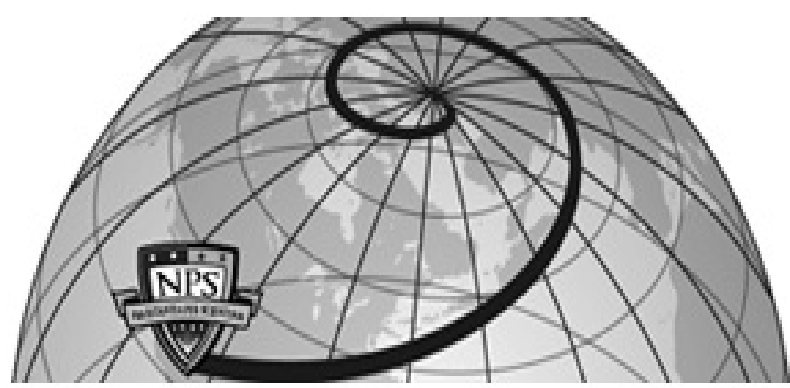

Calhoun: The NPS Institutional Archive DSpace Repository

\title{
A Data Analysis of Success in OCS, The Use of ASVAB Waivers, and Race
}

Read, R.R.; Whitaker, L.R.

Military Operations Research

Military Operations Research, V2 N2 1996

https://hdl.handle.net/10945/38447

This publication is a work of the U.S. Government as defined in Title 17, United States Code, Section 101. Copyright protection is not available for this work in the United States.

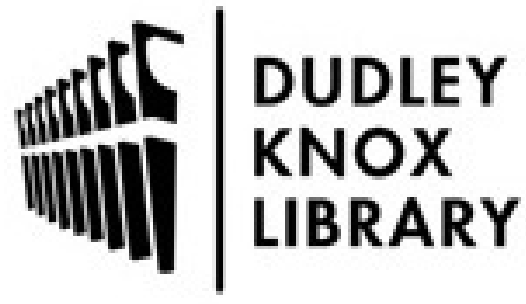

http://www.nps.edu/library
Calhoun is the Naval Postgraduate School's public access digital repository for research materials and institutional publications created by the NPS community. Calhoun is named for Professor of Mathematics Guy K. Calhoun, NPS's first appointed -- and published -- scholarly author.

Dudley Knox Library / Naval Postgraduate School 411 Dyer Road / 1 University Circle Monterey, California USA 93943 


\section{ABSTRACT}

Applicants for Officers Candidate School (OCS) can receive a mental aptitude qualification waiver based upon their scores on the electronics portion of the Armed Services Vocational Aptitude Battery (ASVAB). The question arises whether the candidates that receive a waiver have the same success rate in OCS as those who do not. From OCS records there is strong evidence that the overall rate of success in OCS is smaller for those candidates who hold a waiver than for those candidates who do not hold a waiver. However, closer inspection of the data reveals that success rates change with race in such a way that, for each racial group, the presence or absence of a waiver is not noticeable. That is, success is conditionally independent of waiver. This independence is lost when the conditioning is removed. Thus what initially seemed to be a waiver policy issue is confounded by the rate of granting waivers by race and differences in success rates by race. The OCS data are studied to expose this conundrum and to develop sharper models for success in OCS.

\section{INTRODUCTION}

The accession of officers into the Marine Corps via OCS includes the use of one of three mental aptitude test scores: Armed Services Vocational Aptitude Battery Electronics Repair Composite (ASVAB), the Scholastic Aptitude Test (SAT), and the American College Test (ACT). Historically, $55 \%$ of the officers entering use the first of these three, and the qualification threshold is a score of 120 . But a candidate can receive a waiver of this minimum provided his score is 115 or better. This analysis treats only those using the ASVAB test.

Based on data collected over the fiscal years 1988 through 1992 and broken out by race, personnel at the Manpower Analysis (MA) Branch at Marine Corps Headquarters noticed that success at the Officer Candidate School (OCS) appears to be independent of whether an officer has received an ASVAB waiver. Specifically, there are four recorded racial groups, Caucasian, Black, Hispanic, and Other. The Other group consists largely of American Indian, Alaskan Native, Asian, and Pacific Islander. When collapsed over time, the four $2 \times 2$ contingency table tests for independence yield the chi square test statistics $.6678,2.841, .7983, .5767$ for the respective races, each with one degree of freedom. None of these are significant. However, when the data are further collapsed over race and a single test for independence is performed, then the relationship is highly significant. This latter $2 \times 2$ table appears in Table 1. The chi square statistic is 11.87 and the p-value is 0.00057 .

On the surface, it appears that we have contradictory results. On the one hand, OCS candidate success and the presence of a waiver are independent when Caucasians, Blacks, Hispanics and Others are considered separately. On the other hand, there is dependence in the collapsed table when race is not accounted for, with strong evidence that the chance of success without a waiver $(76 \%)$ is greater than that with a waiver (72\%).

\begin{tabular}{|c|c|c|c|}
\hline & Waiver & No Waiver & Total \\
\hline Success & 754 & 7449 & 8203 \\
\hline Failure & 299 & 2303 & 2602 \\
\hline Total & 1053 & 9752 & 10805 \\
\hline
\end{tabular}

Table 1. Macro Analysis of Success and Waiver

A short answer to the contradiction can be obtained through an interpretation of the two success rates. They are not significantly different for waiver and non-waiver within racial groups. But the rates change sharply from group to group. Indeed, the use of the waiver varies markedly from group to group and, to a lesser extent, from year to year. This is surely related to the implementation of the Marine Corps Affirmative Action Plan.

This paper contains an explanation of the contradiction and attention is drawn to other interesting facets as well. In Section II the raw data are presented and all $2 \times 2$ tables of success/failure by waiver/nonwaiver are studied for each year/racial group pair. Generally, independence is tenable. To explain the non-independence, the full data, aggregated over years and with race as a factor, are then subjected to a loglinear analysis in Section III. In Section IV, we fit models with time as a factor including the use of the waiver by year and race. These models could be valuable because an illadvised long-term overuse of the waiver could lead to inequities in the future advancement to higher rank [3].

\section{A Data} Analysis of Success in OCS, The Use of ASVAB Waivers, and Race

\author{
R.R. Read \\ L.R. Whitaker \\ Naval Postgraduate School
}

Application Areas:

Manpower and Personnel, Training

OR Methodologies:

Statistics, Categorical Data Analysis 
Categorical data is prevalent in military OR. Thus, we take a careful look at the data and provide details that would normally be omitted so that certain usage may be illustrated. In particular, in the next section, attention is drawn to the rather interesting effects when conditional tests are used, and in Section III the steps for fitting a loglinear model are presented.

The factors of interest are success or failure of candidates in the OCS program, whether the candidate used an ASVAB (lower mental category) waiver, fiscal year, and race. The data (see Table 2) consists of counts

$$
D_{i j k l}
$$

where $i=1,2$ indicates success or failure, $j=1,2$ indicates presence or absence of waivers, $k=1, \ldots, 5$ indicates the fiscal year FY88 to FY92 and $l=1, \ldots, 4$ indicates race, in the order given earlier.

\begin{tabular}{c|c|cccc|c|}
\multicolumn{7}{c}{ Candidates Qualifying with ASVAB Waiver } \\
\cline { 2 - 8 } & FY & White & Black & Hispanic & Other & Total \\
\cline { 2 - 8 } Success & FY88 & 100 & 11 & 10 & 12 & 133 \\
in & FY89 & 142 & 37 & 12 & 20 & 211 \\
OCS & FY90 & 102 & 30 & 20 & 11 & 163 \\
& FY91 & 77 & 22 & 14 & 2 & 115 \\
& FY92 & 70 & 36 & 22 & 4 & 132 \\
\hline & Total & 491 & 136 & 78 & 49 & 754 \\
\hline \multirow{5}{*}{ Failure } & FY & White & Black & Hispanic & Other & Total \\
\hline \multirow{5}{*}{ OCS } & FY88 & 22 & 8 & 5 & 1 & 36 \\
& FY89 & 30 & 15 & 11 & 7 & 63 \\
& FY90 & 35 & 16 & 10 & 3 & 64 \\
& FY91 & 21 & 22 & 6 & 3 & 52 \\
& FY92 & 45 & 31 & 8 & 0 & 84 \\
\hline & Total & 153 & 92 & 40 & 14 & 299 \\
\hline
\end{tabular}

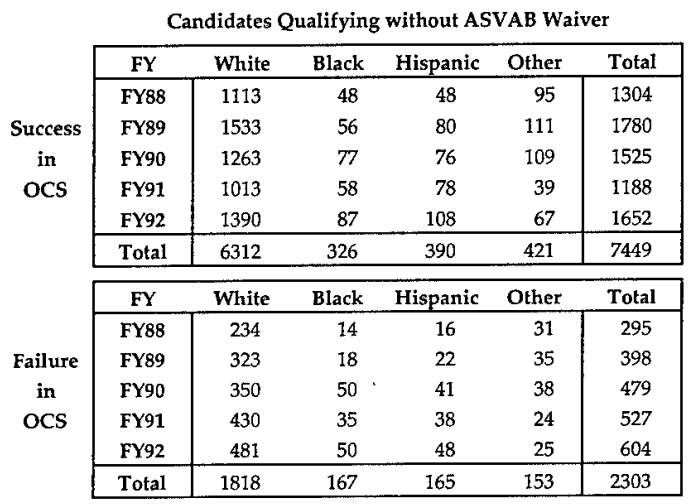

Table 2. Frequency Counts by Category

\section{INDIVIDUAL CONTINGENCY TABLES}

Suppose the full data are broken into twenty (5 years, 4 races) $2 \times 2$ contingency tables and subjected to individual analyses. It is instructive to apply the most often used procedures to each and gain experience in their use and effect.

Let us simplify the notation and let $n_{i j}=D_{i j k l}$ be the counts with year and race held fixed, $i=1,2$ indicates success or failure in OCS, and $j=1,2$ indicates presence or absence of waiver, respectively. Under independence the expected frequencies are estimated by

$$
\hat{m}_{i j}=n_{i+} n_{+j} / N \quad \text { with } N=\sum \sum n_{i j},
$$

and the plus indicates summation over the replaced subscript. The estimated frequencies under independence based on Table 1 are given in Table 3. The estimated success rate is $76 \%$ in both instances. The familiar Pearson Chi Square and Log Likelihood statistics are given by

$$
\begin{aligned}
& \chi^{2}=\sum_{i=1}^{2} \sum_{j=1}^{2}\left(n_{i j}-\hat{m}_{i j}\right)^{2} / \hat{m}_{i j} \\
& G^{2}=2 \sum_{i=1}^{2} \sum_{j=1}^{2} n_{i j} \ln \left(n_{i j} / \hat{m}_{i j}\right)
\end{aligned}
$$

Each is asymptotically distributed as chi square with one degree of freedom.

\begin{tabular}{|c|cc|c|}
\hline \multicolumn{1}{c}{} & Waiver & No Waiver & Total \\
\hline Success & 799 & 7404 & $\mathbf{8 2 0 3}$ \\
Failure & 254 & 2348 & 2602 \\
\hline Total & 1053 & 9752 & 10805 \\
\hline
\end{tabular}

Table 3. Estimate Frequencies under Independence

The use of the odds ratio is also popular especially in $2 \times 2$ tables. It summarizes the strength and type of dependence between the two categories. Letting $\left\{\Pi_{i j}\right\}$ be the cell probabilities, the odds ratio is defined by

$$
\theta=\Pi_{11} \Pi_{22} / \Pi_{12} \Pi_{21}
$$


A DATA ANALYSIS OF SUCCESS IN OCS

and, in our context, represents the odds of OCS success using waivers divided by the odds of success without the use of waivers. The null value $\theta=1$ represents "no effect" of waivers, or independence. The maximum likelihood estimator of $\theta$ is

$$
\hat{\theta}=n_{11} n_{22} / n_{12} n_{21} .
$$

The null distribution of $\ln (\hat{\theta})$ is well approximated by the normal distribution [1] with the variance estimated by

$$
[\hat{\sigma}(\ln \hat{\theta})]^{2}=\sum_{1}^{2} \sum_{1}^{2} 1 / n_{i j} .
$$

Thus, a third test statistic is

$$
Z=\ln (\hat{\theta}) /\left[\sum \sum 1 / n_{i j}\right]^{1 / 2} \text {. }
$$

Concern for the use of asymptotics has led the authors to consider Fisher's Exact Test as well, [1, p60ff]. Under the null hypothesis of independence, an exact distribution that is free of any unknown parameters results from conditioning on the totals in both margins. The result is a hypergeometric distribution

$$
\left(\begin{array}{l}
n_{+1} \\
n_{11}
\end{array}\right)\left(\begin{array}{l}
n_{+2} \\
n_{12}
\end{array}\right) /\left(\begin{array}{l}
N \\
n_{1+}
\end{array}\right) .
$$

Since the totals in the margins are given, only $n_{11}$ need be considered as variable. Its range is

$$
\max \left(0, n_{+1}+n_{1+}-N\right) \leq n_{11} \leq \min \left(n_{+1}, n_{1+}\right) .
$$

Exact two-sided p-values are obtained by summing probabilities of tables that are at least as rare under the null hypothesis as the observed table. Only those tables that have hypergeometric probabilities at least as small as the observed configuration are used [2].

The results of the four procedures are given in Table 4, which contains the values of total populations, $N$; the odds ratios, $\hat{\theta}$; $\ln (\hat{\theta})$; the standard deviation of $\ln (\hat{\theta})$; and the four p-values. Within cells the racial levels are Caucasian, Black, Hispanic, Other, respectively. There are some blank entries for the last case because $n_{21}=0$.

\begin{tabular}{|l|lrrrrrrrr|}
\hline \multicolumn{1}{|c}{} & \multicolumn{1}{c}{$N$} & $\hat{\theta}$ & $\ln \hat{\theta}$ & $\hat{\sigma}(\ln \hat{\theta})$ & $X^{2}$ & $G^{2}$ & $Z$ & Fisher \\
\hline FY88 & Cauc. & 1469 & .956 & -.045 & .246 & .854 & .854 & .854 & .804 \\
& Black & 81 & .401 & -.914 & .555 & .094 & .104 & .100 & .139 \\
& Hisp. & 79 & .667 & -.405 & .619 & .511 & .518 & .513 & .527 \\
& Other & 139 & 3.916 & 1.365 & 1.061 & .168 & .126 & .198 & .298 \\
\hline FY89 & Cauc. & 2028 & .997 & -.003 & .210 & .990 & .990 & .990 & 1.000 \\
& Black & 126 & .793 & -.232 & .409 & .570 & .571 & .570 & .681 \\
& Hisp. & 125 & .300 & -1.204 & .482 & .010 & .014 & .012 & .017 \\
& Other & 173 & .901 & -.104 & .480 & .828 & .829 & .828 & .810 \\
\hline FY90 & Cauc. & 1750 & .808 & -.213 & .205 & .296 & .304 & .297 & .285 \\
& Black & 173 & 1.218 & .197 & .359 & .583 & .582 & .583 & .723 \\
& Hisp. & 147 & 1.079 & .076 & .433 & .861 & .860 & .861 & 1.000 \\
& Other & 161 & 1.278 & .245 & .678 & .717 & .712 & .717 & 1.000 \\
\hline FY91 & Cauc. & 1541 & 1.556 & .442 & .253 & .078 & .070 & .080 & .085 \\
& Black & 137 & .603 & -.506 & .370 & .170 & .172 & .172 & .196 \\
& Hisp. & 136 & 1.137 & .128 & .527 & .808 & .807 & .808 & 1.000 \\
& Other & 68 & .410 & -.892 & .949 & .335 & .342 & .348 & .379 \\
\hline FY92 & Cauc. & 1986 & .538 & -.620 & .198 & .002 & .002 & .002 & .002 \\
& Black & 204 & .667 & -.405 & .303 & .180 & .182 & .181 & .223 \\
& Hisp. & 186 & 1.222 & .200 & .448 & .654 & .651 & .654 & .828 \\
& Other & 96 & & & & .225 & .116 & & .570 \\
\hline
\end{tabular}

Table 4. Two-Sided p-values

Perhaps the first thing to notice is the agreement of $\mathrm{p}$-values for the three asymptotic procedures. Only for the smaller values of $N$ do they show much separation. On the other hand, the p-values for Fisher's Exact Test generally tend to be higher. The main reason for this is the conditioning on both margin totals. Such is not the case in the other procedures. By conditioning on the margin totals, the nuisance parameters are eliminated in Fisher's Exact Test while in the other three procedures they are estimated.

The differences in p-values do not lead to conflicting conclusions, however. Two cases of the twenty are significant: Hispanics ' 89 and Caucasians '92. In both of these cases the odds for success are smaller if waivers are used. The opposite is true for Caucasians '91, a case that might be controversial as $p \sim .08$.

\section{GENERAL MODELS}

The four factors; success / failure, waiver/no waiver, year $(1, \ldots, 5)$, and race $(1, \ldots, 4)$; are denoted as A, B, C, D, respectively. Since the total number of OCS candidates is not fixed, the data $D_{i j k l}$ will be assumed to be generated from an independent Poisson sampling scheme, i.e., $D_{i j k l}$ are independent Poisson random variables with respective parameters $\left(m_{i j k l}\right)$ where $m_{i j k l}=E\left[D_{i j k l}\right]$. To interpret the results given in 
the introduction we first fit a loglinear model to the counts collapsed over years, i.e., to

$$
D_{i j+l}=\sum_{k=1}^{5} D_{i j k l} .
$$

The saturated loglinear model parameterizes

$$
\begin{aligned}
& m_{i j+l}=E\left[D_{i j+l}\right] \text { as } \\
& \begin{aligned}
& \ln m_{i j+l}=\mu+\lambda_{i}^{A}+\lambda_{j}^{B}+\lambda_{l}^{D}+\lambda_{i j}^{A B}+\lambda_{i l}^{A D}+\lambda_{j l}^{B D}+\lambda_{i j l}^{A B D}, \\
& i=1,2 \quad j=1,2 \quad l=1, \ldots, 4,
\end{aligned}
\end{aligned}
$$

with the contrast conventions

$$
\lambda_{1}^{A}=\lambda_{1}^{B}=\lambda_{1}^{D}=\lambda_{1 j}^{A B}=\lambda_{i 1}^{A B}=\ldots=\lambda_{i j 1}^{A B D}=0
$$

and where the $\lambda^{\prime}$ s are the effects and interaction terms corresponding to the variables A, B, D given in the superscript (e.g. $\lambda_{l}^{D}$ is the effect of race and $\lambda_{j l}^{B D}$ is the interaction term for waiver/no waiver and race). Using standard notation [1], this saturated model can be represented as [ABD], i.e., the third order interaction term $\mathrm{ABD}$ and all lower order terms made up of subsets of the variables $A, B$, and D are included in the model. We begin by fitting the model with all two-way interaction terms along with all main effects, i.e., the model $[\mathrm{AB}][\mathrm{AD}][\mathrm{BD}]$. This gives a likelihood ratio test statistic of 2.55 with 3 degrees of freedom and a p-value of .466. This model does fit the data. To see whether a more parsimonious model can be fit we remove twoway interaction terms one at a time. This yields the model [AD] [BD]. The overall likelihood ratio test statistic is 4.84 with 4 degrees of freedom giving an acceptable p-value of .31 . To see whether anything has been lost by removing the $\mathrm{AB}$ interaction term, we test the null hypothesis $[\mathrm{AD}][\mathrm{BD}]$ versus the alternative $[\mathrm{AB}][\mathrm{AD}][\mathrm{BD}]$. The test statistic 1.99 with 1 degree of freedom has a p-value of .256. There is not enough evidence to indicate that the $A B$ term should be included. Further, deleting terms from the [AD] $[\mathrm{BD}]$ model yields models with unacceptable fits, i.e., those with likelihood ratio test statistics having p-values less than .05 . Finally, the standardized residuals for the $[\mathrm{AD}][\mathrm{BD}]$ model range from -.843 to 1.090 . Thus, the model $[\mathrm{AD}][\mathrm{BD}]$ is selected and fits the data (collapsed over years) reasonably well.
The question now becomes, can this model account for the results that motivated the study. The probabilistic interpretation of the model [AD] [BD] is that conditional on the levels of factor $\mathrm{D}$ (race), the variables $\mathrm{A}$ and $\mathrm{B}$ are independent. To see this note that the joint probability mass function (pmf) of the variables $\mathrm{A}, \mathrm{B}, \mathrm{C}, \mathrm{D}$ is

$$
P_{i j k l}=\frac{m_{i j k l}}{m_{++++}},
$$

for $i=1,2 ; j=1,2 ; k=1, \ldots, 5$; and $l=1, \ldots, 4$. The model $[\mathrm{AB}][\mathrm{BD}]$ fitted to the data collapsed over years corresponds to

$\ln m_{i j+l}=\mu+\lambda_{i}^{A}+\lambda_{j}^{B}+\lambda_{l}^{D}+\lambda_{i l}^{A D}+\lambda_{j l}^{B D}$.

Thus the conditional pmf of $A$ given that $B$ is at level $j$ and $D$ is at level $l$ can be found from this model to be

$$
\begin{aligned}
P_{i \mid j l} & =\frac{P_{i j+l}}{P_{+j+l}}, \\
& =\frac{\exp \left\{\mu+\lambda_{i}^{A}+\lambda_{l}^{D}+\lambda_{i l}^{A D}\right\}}{\sum_{i} \exp \left\{\mu+\lambda_{i}^{A}+\lambda_{l}^{D}+\lambda_{i l}^{A D}\right\}} .
\end{aligned}
$$

Since the right hand side of (3.2) is not a function of $j$, we see that the conditional pmf of A given $\mathrm{B}, \mathrm{D}$ is the same as the conditional pmf of $\mathrm{A}$ given $D$. Thus given $D$, the factors $A$ and $B$ are independent.

However, $A$ and $B$ are not independent by themselves alone. The marginal probabilities of these two factors can be developed from the model (3.1) by summing

$$
\exp \left\{\mu+\lambda_{i}^{A}\right\} \sum_{\ell} \sum_{j} \exp \left\{\lambda_{j}^{B}+\lambda_{\ell}^{D}+\lambda_{i \ell}^{A D}+\lambda_{j \ell}^{B D}\right\}
$$

and

$$
\exp \left\{\mu+\lambda_{j}^{B}\right\} \sum_{\ell} \sum_{i} \exp \left\{\lambda_{i}^{A}+\lambda_{\ell}^{D}+\lambda_{i \ell}^{A D}+\lambda_{j \ell}^{B D}\right\}
$$

and forming the appropriate normalizations. The joint probability is not the product of these probabilities. Thus the model supports the observation made earlier that success of the OCS candidate is not independent of whether the ASVAB waiver has been used for entry. These 


\section{A DATA ANALYSIS OF SUCCESS IN OCS}

two variables are independent, however, when broken out by race.

The following probabilities help interpret the dependence between $A$ and $B$. The probabilities of success given race are estimated to be .78 , $.64, .70, .74$ for Caucasians, Blacks, Hispanics and Others, respectively. (The empirical rates and the modeled rates are the same to two decimal places.) Because success is independent of waiver status given race, these probabilities are the same for those candidates with a waiver and those candidates without a waiver. The proportions of candidates in each race which possess a waiver are $.07, .32, .18, .10$, and the proportions of candidates who don't possess a waiver in each race are the complementary values, $.93, .68$, $.82, .90$. The greatest proportion of candidates who don't possess a waiver are Caucasians $(93 \%)$, with a good chance of success $(78 \%)$. However, candidates that do utilize the waiver are divided primarily between Blacks (32\%) and Hispanics (18\%). Because the probability of success for these two races differ $(67 \%)$ and $(70 \%)$ respectively, we see that the overall probability of success with a waiver is lower than without a waiver. Also, the four success rates decrease monotonically as the four waiver use rates increase. Thus the difference in the overall success rate among those who hold a waiver and those who do not does not appear to be caused solely by the presence of waiver but by differences in success rates between races and the differences in the proportions of waivers given by race.

\section{TEMPORAL ANALYSIS}

The above analysis responds to the question posed in the introduction. But it is also of interest to consider the other factor, $\mathrm{C}$, the fiscal year. If including the variable race sheds light on the dependence between having a waiver and success of the OCS candidate, perhaps considering this fourth variable will add to an understanding of this data set.

Perhaps the most direct way to proceed is to consider the most general four factor model that reflects independence of factors $A$ and $B$. In the notation established this would be [ACD] [BCD]. All interactions involving $A$ and $B$ are zero. Doing so produces a likelihood ratio p-value of
.049. This is rather small for our tastes. Study of the residuals reveals two outlier cells: unsuccessful Hispanics with a waiver in FY89 and unsuccessful Caucasians with a waiver in FY92. These two cells belong to the same cases that exhibited low p-values in Table 4.

It appears that the loglinear modeling system must provide for some $A B$ interactive terms. Accordingly we apply the strategy which fits the models with all three way and lower order terms; all two way and lower order terms; and all one way terms. Then the overall model with the fewest terms and an acceptable overall fit is used as a starting point for further deletion of terms within the chosen set. The first model fit was the one with all three way interactions. This gives an overall fit with a p-value of .0387 . However, as terms are deleted the p-value increases and the model [ABC] [BCD] [ACD] gives a slightly higher p-value for overall fit of .0657. Further deletion of terms leads to the model [ABC] [BCD] [AD] with p-value .22.

The fact that the deletion of additional terms appears to improve the fit can be explained by noting the increase in the degrees of freedom. For the model with all three way interaction terms, the likelihood ratio test statistic is 21.95 with 12 degrees of freedom, deleting the ABD term increases degrees of freedom to 15 and the test statistic to 24.01 and the deletion of the ABD term increases the degrees of freedom to 19 and the test statistic to 29.548. Therefore deleting terms does not increase the test statistic very much compared to the gain in degrees of freedom.

Deleting either the $A B C$ or $B C D$ terms from the $[A D][A B C][B C D]$ model results in models with much lower $p$-values for overall goodness of fit and standardized residuals that are of much larger magnitude than those of the [AD] $[\mathrm{ABC}][\mathrm{BCD}]$ model. Since the standardized residuals for this model range between -1.78 to 1.81, this model appears to give an adequate fit. In passing, we note that all $A B$ interactive terms are modest in size.

The estimated probabilities of success given race, waiver status and fiscal year $\left(\hat{p}_{i \mid j k l}\right)$ are plotted against year $(k)$ in Figures 1 and 2. There is a general decrease in the probability of success over time in all four racial groups regardless of waiver status. In fact, when the model [AD] [BD] 


\section{A DATA ANALYSIS OF SUCCESS IN OCS}

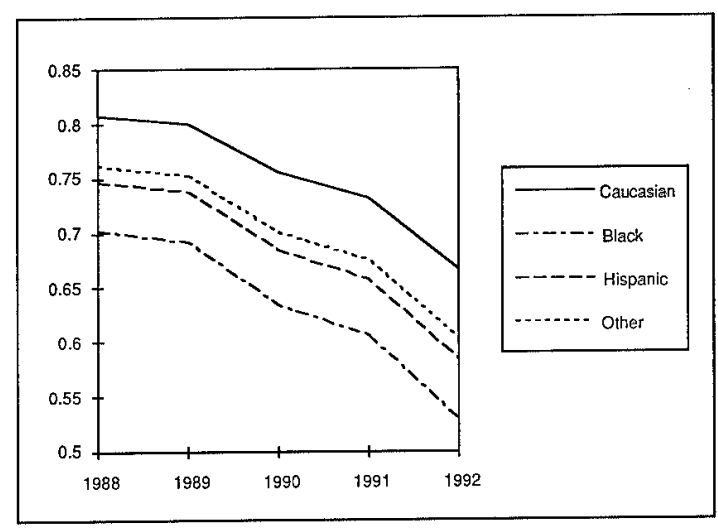

Figure 1. $\hat{P}[S \mid$ with waiver, race, year $]$ vs. Year

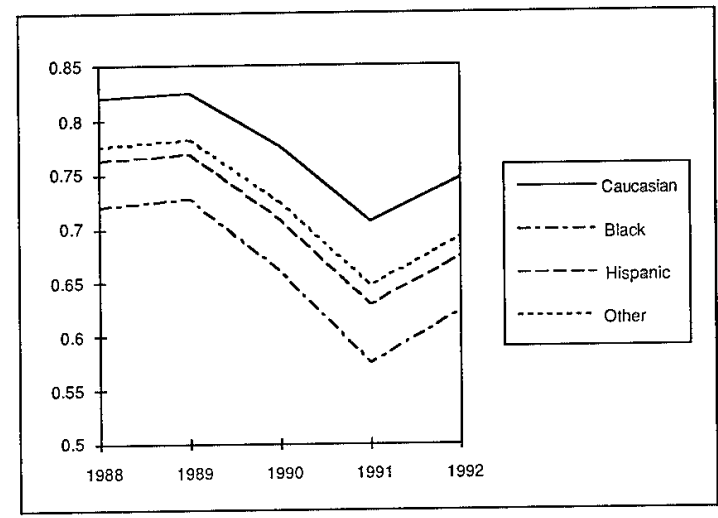

Figure 2.

$\hat{P}[S \mid$ without waiver, race, year] vs. Year

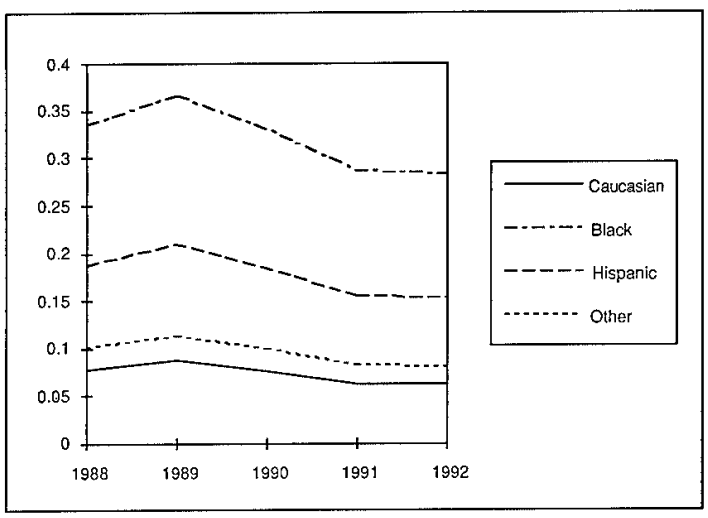

Figure 3.

$\hat{P}$ [granting waiver I race, year] vs. Year

is fit to years separately, only 1992 fails to fit with a $p$-value $=.01$. It appears that for the first four years this trend is reasonably well modeled as independent of waiver status. The presence of the $\mathrm{ABC}$ interaction term in the temporal model is a consequence of changes in 1992, specifically the outlier cell cited earlier.

The presence of the $\mathrm{BCD}$ interaction term can be explained by changes in the number of waivers utilized over time. To examine this, we fit a logistic regression model where the response variable is one or zero according to whether an individual received a waiver or not, and the explanatory variables are years and race. Since years is in fact an ordinal variable, it was scored as the integers 1 to 5 for the years 1988 to 1992. This saves degrees of freedom and helps detect monotonic trends.

The model with a cubic term in years gives an adequate fit to the data ( $\mathrm{p}$-value $=.112$ ). This model fits the data somewhat better than the model that fits the year as a categorical variable.

The fitted values are the estimates of the conditional probabilities that an officer receives a waiver given year and race. These are plotted by race in Figure 3. From this plot it can be seen that except for 1989 there has been a general decline in the proportion of waivers awarded for each race.

In conclusion, we have accounted for the nature of the paradox stated in the introduction by the use of loglinear analysis after collapsing the data over time. The odds ratio analysis served to support the independence vs. waiver hypothesis at a micro-level, and deeper loglinear modeling can be used to quantify the changes in probabilities as functions of race and time. Based on the data and these models, success in OCS has, in general, declined over time for all racial groups independently of waiver status. There does appear to be a marked difference in the probabilities of success among the racial groups. The final analysis collapses the data over OCS success or failure and treats the use of the waiver. It appears to be diminishing in time but there are some rather prominent separations by race. Some additional study in these areas can be found in [3].

For these data, success in OCS (for those qualifying based upon ASVAB test) has declined over time, and is basically independent of waiver status when conditioned on recorded race. Two hypotheses emerge: the ASVAB contains a racial bias that accepts candidates by race group, leading to uneven success rates; or discrimination occurs in OCS, leading to differential success rates despite equal qualification. 


\section{REFERENCES}

[1] Agresti, A. (1990). Categorical Data Analysis, Wiley.

[2] Davis, L. J. (1986). "Exact Tests for $2 \times 2$

Contingency Tables," The American Statistician, Vol. 40, No. 2.

[3] Harrington, D. F. (1993). “An Equal

Opportunity Misconception and the

Accession/Selection Paradox," Marine Corps

Gazette. 